Research Article

\title{
Eigenvalue Criteria for Existence of Positive Solutions to Fractional Boundary Value Problem
}

\author{
Wen Lian $(\mathbb{D})$ and Zhanbing Bai $(1)$ \\ College of Mathematics and Systems Science, Shandong University of Science and Technology, Qingdao 266590, China \\ Correspondence should be addressed to Zhanbing Bai; zhanbingbai@163.com
}

Received 4 March 2020; Accepted 11 April 2020; Published 9 May 2020

Academic Editor: Liguang Wang

Copyright ( 2020 Wen Lian and Zhanbing Bai. This is an open access article distributed under the Creative Commons Attribution License, which permits unrestricted use, distribution, and reproduction in any medium, provided the original work is properly cited.

The existence and multiplicity of positive solutions for the nonlinear fractional differential equation boundary value problem (BVP) ${ }^{C} D_{0+}^{\alpha} y(x)+f(x, y(x))=0, \quad 0<x<1, \quad y(0)=y^{\prime}(1)=y^{\prime \prime}(0)=0$ is established, where $2<\alpha \leq 3,{ }^{C} D_{0+}^{\alpha}$ is the Caputo fractional derivative, and $f:[0,1] \times[0, \infty) \longrightarrow[0, \infty)$ is a continuous function. The conclusion relies on the fixed-point index theory and the Leray-Schauder degree theory. The growth conditions of the nonlinearity with respect to the first eigenvalue of the related linear operator is given to guarantee the existence and multiplicity.

\section{Introduction}

In this paper, we concentrate on the existence and multiplicity of positive solutions for the following problem:

$$
\begin{gathered}
{ }^{C} D_{0+}^{\alpha} y(x)+f(x, y(x))=0, \quad 0<x<1, \\
y(0)=y^{\prime}(1)=y^{\prime \prime}(0)=0,
\end{gathered}
$$

where $2<\alpha \leq 3,{ }^{C} D_{0+}^{\alpha}$ is the Caputo fractional derivative, and $f:[0,1] \times[0,+\infty) \longrightarrow[0,+\infty)$ is a continuous function.

In the past twenty years, the fractional differential equation has aroused great consideration [1-21] not only in its application in mathematics but also in other applications in science and engineering, for example, fluid mechanics, viscoelastic mechanics, electroanalytical chemistry, and biological engineering. Bai and Qiu [22, 23] have investigated the existence and multiplicity of positive solutions of (1) and (2) by using the nonlinear alternative of the Leray-Schauder type and Krasnoselskii's fixed-point theorem in a cone, but they did not consider its eigenvalue criteria.

The rest of the paper is organized as follows. In Section 2, we recall some concepts relative to fractional calculus and give some lemmas with respect to the corresponding Green function. In Section 3, with the use of the fixed-point theory, some existence and multiplicity results of positive solutions are obtained. At last, two examples are given.

\section{Background Materials}

For the convenience of the reader, we give some definitions and lemmas.

Definition 1 (see [23]). The Caputo's fractional derivative of order $\alpha>0$ of a continuous function $y:[0,+\infty) \longrightarrow \mathbb{R}$ is given by

$$
{ }^{C} D_{0+}^{\alpha} y(x)=\frac{1}{\Gamma(n-\alpha)} \int_{0}^{x} \frac{y^{(n)}(t)}{(x-t)^{\alpha-n+1}} d t
$$

where $n-1 \leq \alpha<n$ and $n \in \mathbb{N}$, provided that the right-hand side is pointwise defined on $[0,+\infty)$.

Lemma 2 (see [15]). Given $h \in C[0,1]$, the unique solution of

$$
\begin{gathered}
{ }^{C} D_{0+}^{\alpha} y(x)+h(x)=0, \quad 0<x<1, \\
y(0)=y^{\prime}(1)=y^{\prime \prime}(0)=0,
\end{gathered}
$$


is given by

$$
y(x)=\int_{0}^{1} G(x, t) h(t) d t
$$

where

$G(x, t)=\left\{\begin{array}{l}\frac{(\alpha-1) x(1-t)^{\alpha-2}-(x-t)^{\alpha-1}}{\Gamma(\alpha)}, \quad \text { for } 0 \leq t \leq x \leq 1, \\ \frac{x(1-t)^{\alpha-2}}{\Gamma(\alpha-1)}, \quad \text { for } 0 \leq x \leq t \leq 1,\end{array}\right.$

Lemma 3 (see [23]). The Green function $G(x, t)$ defined by (6) satisfies the following properties:

(i) $G(x, t)>0$, for all $x, t \in(0,1)$

(ii) $\min _{(1 / 4) \leq x \leq(3 / 4)} G(x, t) \geq 1 / 4 \max _{0 \leq x \leq 1} G(x, t)=1 / 4 G(1, t)$, for $t$ $\in(0,1)$

Lemma 4 (see [24]). Let $K$ be a cone in a Banach space $X$, and $\Omega$ be a bounded open set in $K$. Suppose that $T: \bar{\Omega} \longrightarrow K$ is a completely continuous operator. If there exists $y_{0} \in K \backslash\{\theta\}$ such that

$$
y-T y \neq \mu y_{0}, \quad \text { for all } y \in \partial \Omega, \mu \geq 0,
$$

then the fixed-point index $i(T, \Omega, K)=0$.

Lemma 5 (see [24]). Let $K$ be a cone in a Banach space $X$. Suppose that $T: K \longrightarrow K$ is a completely continuous operator. If there exists a bounded open set $\Omega$ such that each solution of

$$
y=\sigma T y, \quad y \in K, \sigma \in[0,1]
$$

satisfies $y \in \Omega$, then the fixed-point index $i(T, \Omega, K)=1$.

Lemma 6 (see [25]). Suppose that $A: C[0,1] \longrightarrow C[0,1]$ is a completely continuous linear operator and $A(K) \subset K$. If there exist $\psi \in C[0,1] \backslash(-K)$ and a constant $c>0$ such that $c$ $A \psi \geq \psi$, then the spectral radius $r(A) \neq 0$ and $A$ has a positive eigenfunction $\varphi^{*}$ corresponding to its first eigenvalue $\lambda_{1}=$ $(r(A))^{-1}$.

\section{Existence and Multiplicity}

Let $C[0,1]$ be endowed with the maximum norm $\|u\|=$ $\max _{0 \leq x \leq 1}|u(x)|$ and the ordering $u \leq v$ if $u(x) \leq v(x)$ for all $x$ $\in[0,1]$. Define

$$
K=\left\{u \in C[0,1] \mid u(x) \geq 0, \min _{(1 / 4) \leq x \leq(3 / 4)} u(x) \geq \frac{1}{4}\|u\|\right\} .
$$

Given $f \in C([0,1] \times[0, \infty),[0, \infty))$. Let $T, A: K \longrightarrow C$ $[0,1]$ be the operators defined by

$$
(T u)(x):=\int_{0}^{1} G(x, t) f(t, u(t)) d t, \quad u \in K, x \in[0,1],
$$

and

$$
(A u)(x):=\int_{0}^{1} G(x, t) u(t) d t, \quad u \in K, x \in[0,1] .
$$

It is well known that $T, A: K \longrightarrow K$ are all completely continuous [23].

Denote

$$
\begin{aligned}
& M=\left(\int_{0}^{1} \max _{0 \leq x \leq 1} G(x, t) d t\right)^{-1}, \\
& N=\left(\int_{(1 / 4)}^{(3 / 4)} G\left(\frac{1}{2}, t\right) d t\right)^{-1},
\end{aligned}
$$

where $M, N$ are positive constants.

Lemma 7. Suppose $A$ is defined by (11), then the spectral radius $r(A)>0$ and $A$ has a positive eigenfunction $\varphi_{1}$ corresponding to its first eigenvalue $\lambda_{1}=(r(A))^{-1}$.

Proof. The operator $A: C[0,1] \longrightarrow C[0,1]$ is a completely continuous linear operator and $A(K) \subseteq K$ (see [23]). Choose $\psi \in C[0,1]$ and $\left[x_{1}, x_{2}\right] \subset(0,1)$ such that $\psi(x) \geq 0$ for $x \in[0,1] ; \psi(x)>0$ for $x \in\left(x_{1}, x_{2}\right)$. By the use of Lemma 2 , for $x \in[0,1]$, there holds

$$
(A \psi)(x)=\int_{0}^{1} G(x, t) \psi(t) d t \geq \int_{x_{1}}^{x_{2}} G(x, t) \psi(t) d t>0 .
$$

So, we can choose $c \in \mathbb{R}$ so large that

$$
c(A \psi)(x) \geq \psi(x), \quad \text { for } x \in[0,1] .
$$

By Lemma 6, we complete the proof.

Theorem 8. Suppose the following conditions hold:

$$
\begin{gathered}
\left(I_{0}\right) \liminf _{y \rightarrow 0^{+}} \frac{f(x, y)}{y}>\lambda_{1}, \\
\left(S_{\infty}\right) \limsup _{y \rightarrow+\infty} \frac{f(x, y)}{y}<\lambda_{1},
\end{gathered}
$$

where $\lambda_{1}$ is the first eigenvalue of the operator $A$ defined by (11). Then, BVP (1) and (2) have at least one positive solution.

Proof. By condition $\left(I_{0}\right)$, there exists $r_{1}>0$ small enough such that

$$
f(x, y) \geq \lambda_{1} y, \quad \text { for all } 0 \leq x \leq 1,0 \leq y \leq r_{1} .
$$


Let $\varphi^{*}$ be the positive eigenfunction of $A$ corresponding to $\lambda_{1}$, thus $\varphi^{*}=\lambda_{1} A \varphi^{*}$.

For every $\varphi \in \overline{K_{r_{1}}}$, for $x \in[0,1]$

$$
\begin{aligned}
(T \varphi)(x) & =\int_{0}^{1} G(x, t) f(t, \varphi(t)) d t \\
& \geq \lambda_{1} \int_{0}^{1} G(x, t) \varphi(t) d t=\lambda_{1}(A \varphi)(x) .
\end{aligned}
$$

Suppose without loss of generality that $T$ has no fixed point on $\partial K_{r_{1}}$ (otherwise, the proof is completed). We claim that

$$
\varphi-T \varphi \neq \mu \varphi^{*}, \quad \text { for all } \varphi \in \partial K_{r_{1}}, \mu>0 .
$$

In fact, if there exist $\varphi_{1} \in \partial K_{r_{1}}$ and $\mu_{0}>0$ such that $\varphi_{1}-T \varphi_{1}=\mu_{0} \varphi^{*}$, then

$$
\varphi_{1}=T \varphi_{1}+\mu_{0} \varphi^{*} \geq \mu_{0} \varphi^{*} .
$$

Let

$$
\mu^{*}=\sup \left\{\mu \mid \varphi_{1} \geq \mu \varphi^{*}\right\} .
$$

It is easy to see that $+\infty>\mu^{*} \geq \mu_{0}>0$ and $\varphi_{1} \geq \mu^{*} \varphi^{*}$. Taking into account that $A$ is a linear positive operator, we have

$$
\lambda_{1} A \varphi_{1} \geq \mu^{*} \lambda_{1} A \varphi^{*}=\mu^{*} \varphi^{*} .
$$

Therefore, by (17),

$$
\varphi_{1}=T \varphi_{1}+\mu_{0} \varphi^{*} \geq \lambda_{1} A \varphi_{1}+\mu_{0} \varphi^{*} \geq\left(\mu^{*}+\mu_{0}\right) \varphi^{*},
$$

which contradicts the definition of $\mu^{*}$. Hence (18) holds and we have from Lemma 3 that

$$
i\left(T, K_{r_{1}}, K\right)=0 .
$$

On the other hand, by $\left(S_{\infty}\right)$, there exist $0<\sigma<1$ and $r_{2}>r_{1}$ such that

$$
f(x, y) \leq \sigma \lambda_{1} y, \quad \text { for all } y \geq r_{2}, x \in[0,1] .
$$

Define $A_{1}: C[0,1] \longrightarrow C[0,1]$ as $A_{1} \varphi=\sigma \lambda_{1} A \varphi, \varphi \in C[0,1]$. Then $A_{1}$ is a bounded linear operator and $A_{1}(K) \subset K$. Denote

$$
B=\left(\max _{0 \leq x, t \leq 1} G(x, t)\right) \sup _{y \in \bar{B}_{r_{2}}} \int_{0}^{1} f(t, y(t)) d t .
$$

It is clear that $B<+\infty$. Let

$$
W=\{\varphi \in K \mid \varphi=\mu T \varphi, 0 \leq \mu \leq 1\} .
$$

In the following, we firstly prove that the set $W$ is bounded.
For any $\varphi \in W$, set $\bar{\varphi}(x)=\min \left\{\varphi(x), r_{2}\right\}$ for $x \in[0,1]$ and denote $E(\varphi)=\left\{x \in[0,1] \mid \varphi(x)>r_{2}\right\}$, then for $x \in[0,1]$,

$$
\begin{aligned}
\varphi(x) & =\mu(T \varphi)(x) \leq(T \varphi)(x)=\int_{0}^{1} G(x, t) f(t, \varphi(t)) d t \\
& =\int_{E(\varphi)} G(x, t) f(t, \varphi(t)) d t+\int_{[0,1] \backslash E(\varphi)} G(x, t) f(t \bar{\varphi}(t)) d t \\
& \leq \int_{0}^{1} G(x, t) \sigma \lambda_{1} \varphi(t) d t+\int_{0}^{1} G(x, t) f(t, \bar{\varphi}(t)) d t \\
& \leq\left(A_{1} \varphi\right)(x)+B .
\end{aligned}
$$

Thus $\left(\left(I-A_{1}\right) \varphi\right)(x) \leq B, x \in[0,1]$. Since $\lambda_{1}$ is the first eigenvalue of $A$ and $0<\sigma<1$, the first eigenvalue of $A_{1}$, $\left(r\left(A_{1}\right)\right)^{-1}>1$. Therefore, the inverse operator $\left(I-A_{1}\right)^{-1}$ exists and

$$
\left(I-A_{1}\right)^{-1}=I+A_{1}+A_{1}^{2}+\cdots+A_{1}^{n}+\cdots .
$$

It follows from $A_{1}(K) \subset K$ that $\left(I-A_{1}\right)^{-1}(K) \subset K$. So we have $\varphi(x) \leq\left(I-A_{1}\right)^{-1} B, x \in[0,1]$ and the set $W$ is bounded.

Choose $r_{3}>\max \left\{r_{2},\left\|\left(I-A_{1}\right)^{-1} B\right\|\right\}$. Then by Lemma 4 , we have

$$
i\left(T, K_{r_{3}}, K\right)=1 .
$$

By (23) and (29), one has

$$
i\left(\frac{T, K_{r_{3}}}{\overline{K_{r_{1}}}, K}\right)=i\left(T, K_{r_{3}}, K\right)-i\left(T, K_{r_{1}}, K\right)=1 .
$$

Then, $T$ has at least one fixed point on $K_{r_{3}} \backslash \overline{K_{r_{1}}}$. This means that problem (1) and (2) have at least one positive solution. The proof is complete.

Theorem 9. Suppose the following conditions are met:

$$
\begin{aligned}
& \left(I_{\infty}\right) \liminf _{y \rightarrow+\infty} \frac{f(x, y)}{y}<\lambda_{1}, \\
& \left(S_{0}\right) \limsup _{y \rightarrow 0^{+}} \frac{f(x, y)}{y}<\lambda_{1},
\end{aligned}
$$

where $\lambda_{1}$ is the first eigenvalue of the operator $A$ defined by (11). Then BVP (1) and (2) have at least one positive solution. The proof is similar to Theorem 8.

Theorem 10. Suppose there exist two numbers $b>a>0$ such that the following conditions are met:

$\left(C_{1}\right) f(x, y) \leq M a, \quad$ for $0 \leq x \leq 1$ and $0 \leq y \leq a$,

$\left(C_{2}\right) f(x, y) \geq N b, \quad$ for $\frac{1}{4} \leq x \leq \frac{3}{4}$ and $\frac{1}{4} b \leq y \leq b$.

Then, BVP (1) and (2) have at least one positive solution. 
Proof. If $\mathrm{C}_{1}$ and $\mathrm{C}_{2}$ hold, similar to Lemma 3 [6], we have

$$
\begin{aligned}
& i\left(A, K_{a}, K\right)=1, \\
& i\left(A, K_{b}, K\right)=0 .
\end{aligned}
$$

Consequently, the additivity of the fixed-point index implies

$$
i\left(\frac{A, K_{b}}{\bar{K}_{a}, K}\right)=i\left(A, K_{b}, K\right)-i\left(A, K_{a}, K\right)=-1
$$

Consequently, $A$ has a fixed point $y(x)$ in $K_{b} \backslash \bar{K}_{a}$.

Theorem 11. The problem in (1) and (2) has at least two positive solutions if conditions $\left(I_{0}\right),\left(I_{\infty}\right)$, and $C_{1}$ hold, where $\lambda_{1}$ is the first eigenvalue of the operator $A$ defined by (11).

Proof. Because $\left(I_{0}\right)$ and $\left(I_{\infty}\right)$ hold, there exist $0<r<a<R$ such that

$$
\begin{aligned}
i\left(A, K_{r}, K\right) & =0, \\
i\left(A, K_{R}, K\right) & =0 .
\end{aligned}
$$

On the other hand, $\mathrm{C}_{1}$ implies $i\left(A, K_{a}, K\right)=1$. So we have

$$
\begin{aligned}
& i\left(\frac{A, K_{a}}{\bar{K}_{r}, K}\right)=i\left(A, K_{a}, K\right)-i\left(A, K_{r}, K\right)=1, \\
& i\left(\frac{A, K_{R}}{\bar{K}_{a}, K}\right)=i\left(A, K_{R}, K\right)-i\left(A, K_{a}, K\right)=-1,
\end{aligned}
$$

therefore, $A$ has two fixed points, $y_{1} \in K_{a} \backslash \bar{K}_{r}, y_{2} \in K_{R} \backslash \bar{K}_{a}$.

Theorem 12. The problem in (1) and (2) has at least two positive solutions if conditions $\left(S_{0}\right),\left(S_{\infty}\right)$, and $C_{2}$ hold, where $\lambda_{1}$ is the first eigenvalue of the operator $A$ defined by (10).

Proof. Because $\left(S_{0}\right)$ and $\left(S_{\infty}\right)$ hold, there exist $0<r<b<R$ such that

$$
\begin{aligned}
& i\left(A, K_{r}, K\right)=1, \\
& i\left(A, K_{R}, K\right)=1 .
\end{aligned}
$$

On the other hand $\mathrm{C}_{2}$ implies $i\left(A, K_{b}, K\right)=0$. So we have

$$
\begin{aligned}
& i\left(\frac{A, K_{b}}{\bar{K}_{r}, K}\right)=i\left(A, K_{b}, K\right)-i\left(A, K_{r}, K\right)=-1, \\
& i\left(\frac{A, K_{R}}{\bar{K}_{b}, K}\right)=i\left(A, K_{R}, K\right)-i\left(A, K_{b}, K\right)=1 .
\end{aligned}
$$

Therefore, $A$ has two fixed points, $y_{1} \in K_{b} \backslash \bar{K}_{r}, y_{2} \in K_{R} \backslash \bar{K}_{b}$.

\section{Example}

To illustrate the main points, we give two examples.
Example 13. Let

$$
f(x, y)=y^{(1 / 2)}+\frac{2+\sin x}{4} .
$$

Consider the BVP

$$
\begin{gathered}
{ }^{C} D_{0+}^{\alpha} y(x)+[y(x)]^{(1 / 2)}+\frac{2+\sin x}{4}=0, \quad 0<x<1, \\
y(0)=y^{\prime}(1)=y^{\prime \prime}(0)=0 .
\end{gathered}
$$

It is not difficult to see that

$$
\frac{f(x, y)}{y}=y^{-(1 / 2)}+\frac{2+\sin x}{4 y}
$$

Then

$$
\begin{aligned}
& \liminf _{y \rightarrow 0^{+}} \frac{f(x, y)}{y}=+\infty>\lambda_{1}, \\
& \limsup _{y \rightarrow+\infty} \frac{f(x, y)}{y}=0<\lambda_{1},
\end{aligned}
$$

where $\lambda_{1}$ is the first eigenvalue of the operator $A$ defined by (11). By Theorem 11, BVP (40) and (41) have at least one positive solution.

Example 14. Let

$$
f(x, y)=y^{3}+y^{2} \sin x
$$

Consider the BVP

$$
\begin{gathered}
{ }^{C} D_{0+}^{\alpha} y(x)+[y(x)]^{3}+[y(x)]^{2} \sin x=0, \quad 0<x<1, \\
y(0)=y^{\prime}(1)=y^{\prime \prime}(0)=0 .
\end{gathered}
$$

It is not difficult to see that

$$
\frac{f(x, y)}{y}=y^{2}+y \sin x
$$

Then

$$
\begin{aligned}
& \liminf _{y \rightarrow+\infty} \frac{f(x, y)}{y}=+\infty>\lambda_{1}, \\
& \limsup _{y \rightarrow+0^{+}} \frac{f(x, y)}{y}=0<\lambda_{1},
\end{aligned}
$$

where $\lambda_{1}$ is the first eigenvalue of the operator $A$ defined by (11). By Theorem 12, BVP (45) and (46) have at least one positive solution.

\section{Data Availability}

Data sharing not applicable to this article as no datasets were generated or analysed during the current study. 


\section{Conflicts of Interest}

The authors declare that there is no conflict of interest regarding the publication of this paper.

\section{Authors' Contributions}

All authors contributed equally to the writing of this paper. All authors read and approved the final manuscript.

\section{Acknowledgments}

This work is supported by NSFC (11571207) and the Taishan Scholar Project.

\section{References}

[1] Z. Bai, Y. Cheng, and S. Sun, "On solutions of a class of threepoint fractional boundary value problems," Boundary Value Problems, vol. 2020, no. 1, 2020.

[2] Z. Bai, Z. Du, and S. Zhang, "Iterative method for a class of fourth-order $p$-Laplacian beam equation," Journal of Applied Analysis and Computation, vol. 9, no. 4, pp. 1443-1453, 2019.

[3] Z. Bai and Y. Zhang, "Solvability of fractional three-point boundary value problems with nonlinear growth," Applied Mathematics and Computation, vol. 218, no. 5, pp. 17191725, 2011.

[4] Y. Cui, "Uniqueness of solution for boundary value problems for fractional differential equations," Applied Mathematics Letters, vol. 51, pp. 48-54, 2016.

[5] Y. Cui, L. Liu, and X. Zhang, "Uniqueness and existence of positive solutions for singular differential systems with coupled integral boundary value problems," Abstract and Applied Analysis, vol. 2013, Article ID 340487, 9 pages, 2013.

[6] L. Erbe, "Eigenvalue criteria for existence of positive solutions to nonlinear boundary value problems," Mathematical and Computer Modelling, vol. 32, no. 5-6, pp. 529-539, 2000.

[7] M. Guo, C. Fu, Y. Zhang, J. Liu, and H. Yang, "Study of ionacoustic solitary waves in a magnetized plasma using the three-dimensional time-space fractional Schamel-KdV equation," Complexity, vol. 2018, Article ID 6852548, 17 pages, 2018.

[8] H. Li and J. Sun, "Positive solutions of superlinear semipositone nonlinear boundary value problems," Computers \& Mathematcs with Applications, vol. 61, no. 9, pp. 2806-2815, 2011.

[9] W. Lian, Z. Bai, and Z. Du, "Existence of solution of a threepoint boundary value problem via variational approach," Applied Mathematics Letters, vol. 104, p. 106283, 2020.

[10] X. Liu and M. Jia, "Solvability and numerical simulations for BVPs of fractional coupled systems involving left and right fractional derivatives," Applied Mathematics and Computation, vol. 353, pp. 230-242, 2019.

[11] X. Liu and M. Jia, "The method of lower and upper solutions for the general boundary value problems of fractional differential equations with $p$-Laplacian," Advances in Difference Equations, vol. 2018, 15 pages, 2018.

[12] Y. Tian, Y. Wei, and S. Sun, "Multiplicity for fractional differential equations with $p$-Laplacian," Boundary Value Problems, vol. 2018, no. 1, 2018.
[13] P. Wang and X. Liu, "Rapid convergence for telegraph systems with periodic boundary conditions," Journal of Function Spaces, vol. 2017, Article ID 1982568, 10 pages, 2017.

[14] Z. Wang, "A numerical method for delayed fractional-order differential equations," Journal of Applied Mathematics, vol. 2013, Article ID 256071, 7 pages, 2013.

[15] Y. Wei and Z. Bai, "Solvability of some fractional boundary value problems with a convection term," Discrete Dynamics in Nature and Society, vol. 2019, Article ID 1230502, 6 pages, 2019.

[16] Y. Wei, Z. Bai, and S. Sun, "On positive solutions for some second-order three-point boundary value problems with convection term," Journal of Inequalities and Applications, vol. 2019, no. 1, 2019.

[17] J. Xu, Z. Wei, D. O'Regan, and Y. Cui, "Infinitely many solutions for fractional Schrodinger-Maxwell equations," Journal of Applied Analysis and Computation, vol. 2019, no. 9, pp. 1165-1182, 2019.

[18] X. Zhang, L. Liu, Y. Wu, and Y. Cui, "Entire blow-up solutions for a quasilinear $p$-Laplacian Schrödinger equation with a nonsquare diffusion term," Applied Mathematics Letters, vol. 74, pp. 85-93, 2017.

[19] Y. Zhang, "Existence results for a coupled system of nonlinear fractional multi-point boundary value problems at resonance," Journal of Inequalities and Applications, vol. 2018, no. 1, 2018.

[20] Q. Zhong, X. Zhang, X. Lu, and Z. Fu, "Uniqueness of successive positive solution for nonlocal singular higher-order fractional differential equations involving arbitrary derivatives," Journal of Function Spaces, vol. 7, 9 pages, 2018.

[21] M. Zuo, X. Hao, L. Liu, and Y. Cui, "Existence results for impulsive fractional integro-differential equation of mixed type with constant coefficient and antiperiodic boundary conditions," Boundary Value Problems, vol. 2017, no. 1, 2017.

[22] Z. Bai and T. Qiu, "Existence of positive solution for singular fractional differential equation," Applied Mathematics and Computation, vol. 215, no. 7, pp. 2761-2767, 2009.

[23] T. Qiu and Z. Bai, "Positive solutions for boundary value problem of nonlinear fractional differential equation," Journal of Nonlinear Sciences and Applications, vol. 1, no. 3, pp. 123131, 2008.

[24] M. A. Krasnosel'skii, Positive Solutions of Operator Equations, Noordhoff, Gronignen, 1964.

[25] D. Guo and J. Sun, Nonlinear Integral Equations, Shandong Science and Technology Press, Jinan, 1985. 